\title{
Intergenerationaler Austausch von Unterstützung und Reziprozität im Kulturvergleich
}

\section{Beate Schwarz \& Gisela Trommsdorff}

\section{$1 \quad$ Einleitung}

Die rapiden demographischen Verănderungen in den Industrieländern, wie zunehmende Lebenserwartung und sinkende Geburtenraten mit der damit verbundenen so genannten „Überalterung“ der Gesellschaft, haben Auswirkungen auf der Makro- wie Mikroebene von Gesellschaften, etwa bei Fragen der Alterssicherung. Die Veränderungen erweisen sich auch für den Einzelnen bzw. die einzelne Familie als bedeutsam. So fuhrt die zunehmende Lebenserwartung dazu, dass Eltern und Kinder noch nie so viel gemeinsame Lebenszeit miteinander verbracht haben wie gegenwärtig (Lauterbach 1995). Da zudem alte Menschen uber einen langen Zeitraum hinweg relativ gesund bleiben (Bundesministerium für Familie, Senioren, Frauen und Jugend 2001), erweitert sich die Zeit, die Eltern und Kinder bei guter Gesundheit, also nicht in der Pflegesituation, miteinander verbringen können. Damit rückt die Frage in den Vordergrund, wie die Eltern-Kind-Beziehung über diesen verlängerten Zeitraum und in dieser spezifischen Lebensphase gestaltet wird.

Die bislang eher deskriptive Forschung zu Eltern-Kind-Beziehungen im Erwachsenenalter liefert mittlerweile ein recht gut abgesichertes Bild, vor allem zu strukturellen Aspekten der Beziehung. Danach pflegen Kinder auch im Erwachsenenalter hăufige Kontakte mit den Eltern. Im Allgemeinen ist die Beziehung erwachsener Kinder und ihrer Eltern gut und eng; es wird sowohl emotionale, praktische und finanzielle Hilfe ausgetauscht. Als besonders eng und vertrauensvoll erweist sich in vielen Studien die Mutter-Tochter-Beziehung (im Uberblick Lye 1996; Mancini \& Blieszner 1989).

Der Deutsche Alterssurvey zeigt, dass fast $70 \%$ der erwachsenen Kinder mehrmals in der Woche Kontakt mit ihren Eltern haben (Szydlik 2000). Dies wird auch dadurch erleichtert, dass die erwachsenen Kinder meist nicht sehr weit von ihren Eltern entfernt wohnen, die Hälfte lebt in der gleichen Stadt oder dem gleichen Bezirk (Kohli \& Künemund 2001; Lauterbach \& Pillemer 2001). 
Aufgrund der zurzeit guten finanziellen Lage vieler älterer Menschen fließt mehr finanzielle Unterstützung von der älteren zur mittleren Generation, bei praktischer Unterstützung ist das Verhältnis eher umgekehrt (Kohli \& Künemund 2001).

Gegenseitige Unterstützung von erwachsenen Kindern und ihren Eltern ist als ein zentraler Aspekt der Beziehung sowohl aus entwicklungspsychologischer Sicht über die Lebensspanne als auch aus soziologischer Sicht von Bedeutung. Es sollte deshalb geklärt werden, welche Bedingungen intergenerationale Unterstützung beeinflussen und wie die Unterstützung mit anderen Beziehungsaspekten zusammenhăngt. Das Modell der ,intergenerationalen Solidarität" von Bengtson und Kollegen (z. B. Bengtson \& Roberts 1991) geht davon aus, dass Normen familiärer Verpflichtung und die emotionale Qualität der Beziehung wichtige Einflussfaktoren für das Ausmaß der intergenerationalen Unterstützung sind. Weiterhin wird in diesem Modell der Austausch von Unterstützung differenziert betrachtet. Unterschieden wird zwischen dem Ausmaß an Unterstützung, die gegeben wird und die man erhält, und der Reziprozität dieses Austausches. Es wird postuliert, dass eine gute emotionale Qualität der Beziehung die Unterstützungen verstärkt und dass Reziprozität im Austausch dieser Unterstützung die Beziehungsqualität verbessert.

Mit dem Konzept der Reziprozităt knüpfen Bengtson und Kollegen an die austauschtheoretischen Überlegungen von Gouldner (1960) an. Danach ist die Stabilität von sozialen Beziehungen davon abhängig, dass die interagierenden Personen sich darauf verlassen können, die von ihnen in die Beziehung eingebrachten Leistungen von dem jeweils anderen in vergleichbarer Weise und innerhalb eines angemessenen Zeitraumes „zurückgezahlt“ zu bekommen. Die postulierte Norm der Reziprozität wird danach auch auf Familienbeziehungen angewendet (z. B. Alt 1994; Schulz 1996), obwohl diese anders als nicht verwandtschaftliche Beziehungen schwer auflösbar sind. Ein "quit pro quo" ist allerdings nicht unbedingt anzunehmen; auch kann eine Art ,langfristiger Vorratshaltung" (support banks) bestehen, durch die Leistungen erst zu einem späteren Zeitpunkt wieder gutgemacht werden (Antonucci 1985).

Die hier beschriebenen theoretischen Konzepte wurden in westlichen Kulturen entwickelt und bisher auch nur dort überprüft. Inwieweit sie auch in nicht westlichen Kulturen Gültigkeit haben, ist eine offene Frage. Gouldner (1960) sah die Norm der Reziprozität als universell gültig an, doch ging er davon aus, dass die Erfüllung dieser Norm kulturspezifische Formen annehmen kann. Bengtson (2001) räumte ein, dass eine kulturvergleichende Überprüfung seines Modells intergenerationaler Solidarität noch weitgehend aussteht. $\mathrm{Zu}$ prüfen 
wäre, ob die in einer Kultur vorherrschenden Werte und Normen, die in beiden theoretischen Ansätzen eine Rolle spielen, die Ausprägung und Funktion der Eltern-Kind-Beziehungen beeinflussen (Trommsdorff \& Kornadt 2003). Dafür erforderliche kulturvergleichende Studien könnten die Ansätze empirisch fundieren und theoretisch weiterentwickeln. Bisher sind jedoch Eltern-KindBeziehung im Erwachsenenalter kaum Gegenstand kulturvergleichender Forschung (Trommsdorff, in press).

\section{Das konfuzianische Konzept der „filial piety“}

Kulturvergleichende Studien sollten kulturangemessene Methoden einsetzen und von einem kulturinformierten Standpunkt aus erfolgen, wobei auch indigene Konzepte der untersuchten Kulturen berücksichtigt werden sollten (Trommsdorff 2003). Für ostasiatische Kulturen ist als indigenes Konzept des Konfuzianismus die „filial piety“ von großer Bedeutung. Damit werden die Rollen und Aufgaben aller Familienmitglieder, besonders aber der Kinder gegenüber ihren Eltern umschrieben. Dass die Familienmitglieder wechselseitig voneinander abhängig sind, ist Voraussetzung für ein hohes Investment der Eltern in ihre Kinder und dafür, dass sich die Kinder wiederum als gehorsam und respektvoll gegenüber ihren Eltern erweisen. Dabei ist die Wahrung der Harmonie in der Familie ein besonders hoher Wert. Insbesondere ist der älteste Sohn verpflichtet, später seine alten Eltern emotional, materiell und spirituell zu unterstützen. Somit ist anzunehmen, dass wie in westlichen ähnlich auch in konfuzianischen Kulturen den familienorientierten Normen und emotionalen Bindungen eine wichtige Rolle für die intergenerationale Unterstützung zukommt. Allerdings sollten in konfuzianischen Kulturen die familienbezogenen Normen stärker als in westlichen Kulturen ausgeprägt sein. Weiter sollte aufgrund der stärkeren gegenseitigen Abhängigkeit auch der Austausch an Unterstützungen intensiver ausfallen.

Das traditionelle Konzept der „filial piety“ hat durch den sozialen Wandel und damit erfolgende Änderungen der Gesellschaftsstruktur einige Veränderungen erfahren, insbesondere was die Rollen des erwachsenen Sohnes und der erwachsenen Tochter angeht. Traditionell beziehen sich die filialen Pflichten auf den ältesten Sohn. Jedoch zeigen aktuelle empirische Studien aus Korea und China, dass zunehmend auch erwachsenen Töchter ihre alten Eltern unterstützen und ein enges emotionales Band zwischen Tochter und Eltern besteht (Levande, Herrick \& Sung 2000; Sun 2002). Diese Ergebnisse werden auch von Nauck 
und Suckow (2003) bestätigt. Die Autoren stellen dazu fest: „Dieser Befund ist deshalb besonders bemerkenswert, als es sich hierbei um verheiratete Töchter handelt und die patrilineare Deszendenz in diesen Gesellschaften offenbar die Erwartung an und das tatsächliche Ausmaß der geleisteten Hilfeleistungen auch nach deren Einheirat in eine andere Lineage nicht vermindert oder unterbricht" (Nauck \& Suckow 2003: 9).

Im Konzept der "filial piety" wird ăhnlich wie in westlichen Kulturen, eine Norm der Reziprozität formuliert: Die Kinder sind verpflichtet, das hohe Investment ihrer Eltern später wieder gutzumachen. Aufgrund der langfristigen zeitlichen Orientierung im Konfuzianismus und der Kontinuităt der Familie uber die Generationen hinweg besteht in den konfuzianischen Kulturen anders als in westlichen Kulturen allerdings für das Kind die Möglichkeit, die „Schuld“ an die Eltern durch eigene Investitionen in die năchste Generation abzutragen, also nicht nur direkt den eigenen Eltern „zurückzuzahlen“ (Hwang 1999). Damit hat die Reziprozitätsnorm in konfuzianischen Kulturen eine andere Bedeutung als in westlichen Kulturen, wo Reziprozităt in Beziehungen auf zeitlich năherem Austausch beruht.

Vor dem Hintergrund der hier skizzierten theoretischen Ansătze und Befunde sollen im Folgenden zwei zentrale Fragen beantwortet werden. (1) Unterscheidet sich das Ausmaß an intergenerationaler Unterstultzung in Deutschland von dem in konfuzianischen Kulturen und wird dies durch Unterschiede in den familienorientierten Normen erklärt? (2) Unterscheidet sich die Bedeutung von Reziprozităt in diesen Kulturen?

\section{Die Studie „Value of Children and Intergenerational Relationships"}

Zur Beantwortung dieser Forschungsfragen dient die Studie " Value of Children and Intergenerational Relationships" Ansătze zum Wert des Kindes und zu den Generationenbeziehungen kulturvergleichend untersucht werden (z. B. Nauck 2001; Trommsdorff 2001; in press). In China, Deutschland, Indonesien, Israel, Südkorea und der Türkei wurden jeweils 300 Mütter sowie eines ihrer Kinder im Jugendalter und in wenigstens

Die „Value of Children“-Studie (VOC-Studie) ist ein von der Deutschen Forschungsgemeinschaft gefordertes Projekt (Na 164/9-1 bis -3, Tr 169/9-1 bis -3). Leitung: Bernhard Nauck, TU Chemnitz und Gisela Trommsdorff, Universitat Konstanz. 
100 Făllen auch die Großmütter mütterlicherseits befragt. Zusätzlich wurden 300 Mütter von Kleinkindern interviewt. Die hier beschriebenen Befunde beruhen auf den Daten der Mutter mit jugendlichen Kindern (im Folgenden: die erwachsenen Töchter) und den Großmüttern (im Folgenden: die Mütter) aus China und Korea als zwei konfuzianisch geprägten Kulturen und aus Deutschland als westliche Kultur. Die Befragungen der Töchter und Mütter erfolgte anhand von standardisierten Fragebögen, die in China und Deutschland durch trainierte Interviewerinnen bei den Befragten zuhause durchgefuhrt wurden. In Korea fullten Tőchter und Mütter die Fragebőgen eigenständig aus.

\subsection{Normen und Werte, die Qualität der Beziehung und der Austausch von Unterstultzung zwischen erwachsenen Töchtern und ihren Müttern ${ }^{2}$}

Angesichts der hohen gegenseitigen Verpflichtungen in den konfuzianischen Kulturen gingen wir von der Annahme aus, dass der Austausch von Unterstultzung zwischen koreanischen und chinesischen Töchtern und ihren Eltern intensiver ist als bei deutschen Tochtern und ihren Eltern. Zur Überprüfung dieser Annahme wurden die Angaben der Tochter verwendet, wie hăufig sie emotionale Unterstützung ( 3 Items; Bsp.: „Wie oft haben Sie in den letzten 12 Monaten versucht, Ihre Eltern trösten?"), instrumentelle Unterstützung (3 Items; Bsp.: "Wie oft haben Sie Ihren Eltern in den letzten 12 Monaten Arbeiten abgenommen, wie Einkaufen oder Arbeiten im Haushalt?") und finanzielle Unterstutzung (1 Item: „Teilen Sie mir bitte mit, wie oft Sie Ihre Eltern in den letzten 12 Monaten finanziell unterstützt haben.") gegeben haben (Antwortskala für alle Items: $1=$ nie bis $5=$ immer $){ }^{3}$ Parallel dazu wurden auch die Mütter nach der Häufigkeit gefragt, zu der sie emotionale, instrumentelle und finanzielle Unterstützung gegeben haben.

2 Die in diesem Abschnitt geschilderten Befunde wurden teilweise auf der ISSBD-Konferenz in Gent (Schwarz, Trommsdorff, Mayer \& Albert 2004) vorgestellt. Die Analysen beruhen auf 207 Mutter-Tochter-Dyaden aus China, 131 Dyaden aus Korea und 100 Dyaden aus Deutschland. Die erwachsenen Tochter waren im Schnitt etwa 40 Jahre alt, die Mutter fast 70 Jahre alt.

3 Genauere Angaben uber die Herkunft sowie der komplette Wortlaut der Items sind zu finden bei Schwarz, Chakkarath, Trommsdorff, Schwenk \& Nauck (2001). Gütekriterien der Skalen für die deutsche Stichprobe sind Mayer, Albert, Schwarz \& Trommsdorff (2003) zu entnehmen. 
Tatsächlich berichten die koreanischen und chinesischen Töchter häufiger, ihren Eltern instrumentelle und finanzielle Unterstützung zu geben als die deutschen Töchter (vgl. Abbildung 1). In Hinblick auf die emotionale Unterstützung gleichen sich koreanische und deutsche Töchter, doch zeigen die chinesischen Töchter häufiger emotionale Unterstützung. Die Mütter aus Korea und China berichten durchweg häufiger emotionale, instrumentelle und finanzielle Unterstützung als die deutschen Mütter.

Abbildung 1: Kulturunterschiede im Ausmaß der Unterstützung, die erwachsene Töchter ihren Eltern geben

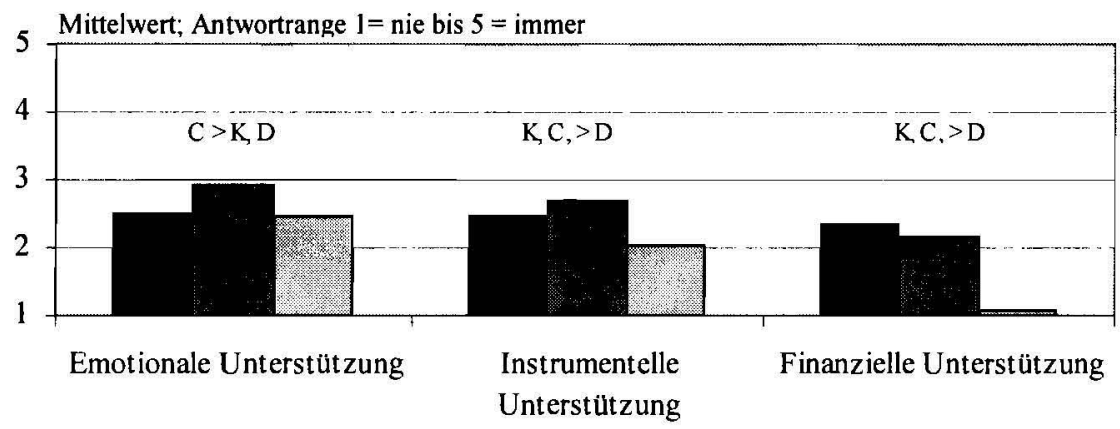

Korea China Deutschland

Datenbasis: $\quad$ VOC-Studie 2002

Es stellte sich nun die Frage, wodurch diese deutlichen kulturellen Unterschiede in der intergenerationalen Unterstützung erklärt werden können. Wie eingangs skizziert, war die Erwartung, dass die höhere gegenseitige Verpflichtung und Abhängigkeit in den Familien den intensiveren Austausch von Unterstützung erklärt. Die Töchter wurden gefragt, welche Erwartungen sie an erwachsene Kinder haben (14 Items; Bsp.: „,dass er/sie weiterhin in Ihrer Nähe lebt“; ,dass er/sie Sie finanziell unterstützt"). Darüber hinaus wurden Mütter und Töchter zu ihren independenten und interdependenten Orientierungen befragt. D. h. sie schätzten ein, inwieweit ihr Selbstkonzept durch persönliche Attribute definiert ist oder durch ihre Zugehörigkeit zu und Anpassung an die Familie (Markus \& Kitayama 1991). Die Skala Interdependenz erwies sich bei Töchtern und Müt- 
tern als reliabel ( 5 Items; Bsp.: „Es ist mir wichtig, Entscheidungen der Familie zu respektieren."), die Skala Independenz jedoch nur bei den Müttern (5 Items, Bsp.: „Es gefällt mir, mich in vielerlei Hinsicht von den anderen Familienmitgliedern zu unterscheiden.").

Die chinesischen Töchter berichten von höheren filialen Erwartungen als die Koreanerinnen, die wiederum höhere Erwartungen als die deutschen Töchter haben. Die chinesischen Töchter erweisen sich als interdependenter orientiert als die koreanischen und deutschen Töchter, die sich in dieser Hinsicht nicht unterscheiden. Bei den Müttern ergeben sich unerwartete Befunde: Die koreanischen Mütter sind sowohl weniger independent als auch weniger interdependent als die chinesischen und deutschen Mütter, die sich nicht unterscheiden.

In hierarchischen Regressionsanalysen zur Vorhersage der Unterstützung durch die Töchter wurde in einem ersten Schritt die Variable Kultur eingeführt, in einem zweiten als Mediatoren des Kultureffekts die Variablen filiale Erwartungen und Interdependenz der Töchter (siehe Tabelle 1). Die häufigere emotionale und instrumentelle Unterstützung, die chinesische Töchter ihren Eltern im Vergleich zu den deutschen Töchtern geben, wird teilweise durch deren stärkere interdependente Orientierung erklärt. Die im Vergleich zu deutschen Töchtern höheren filialen Erwartungen der chinesischen und koreanischen Töchter erklären darüber hinaus einen Teil der häufigeren instrumentellen Unterstützung in diesen beiden Kulturen. Die deutlich häufigere finanzielle Unterstützung der chinesischen und koreanischen Töchter an ihre Eltern wird aber so gut wie gar nicht durch deren Normen und Werte erklärt. Damit sind unsere Erwartungen nur teilweise in Hinblick auf die emotionale und instrumentelle Unterstützung der Töchter an ihre Eltern bestätigt worden.

Bei den Müttern war die Überprüfung vermittelnder Prozesse der Kulturunterschiede in der Unterstützung an die Töchter beschränkt auf die Independenz der Mütter. Doch erklärt eine geringere Independenz nicht die hăufigeren Unterstützungsleistungen koreanischer Mütter an die Töchter. In der Befragung der Mütter fehlen vermutlich die angemessenen psychologischen Indikatoren für familiäre Normen und Werte. Zum Beispiel wurden die filialen Erwartungen der Mütter nicht erfragt.

Sowohl im Konzept der „filial piety“ wie auch im Modell intergenerationaler Solidarität spielt die emotionale Qualität der Beziehung zwischen erwachsenen Kindern und ihren Eltern eine wichtige Rolle. In einem letzten Schritt der Regression wurden deshalb drei Indikatoren der Beziehungsqualität eingeführt, die nicht im Sinne von Mediatoren sondern als additive Effekte geprüft wurden: Intimität in der Beziehung zur Mutter (3 Items; Bsp.: „Wie oft erzählen Sie Ihrer 
Mutter alles, was Sie beschäftigt?"), perzipierte Wertschätzung durch die Mutter (3 Items; Bsp.: „Wie oft lăsst Ihre Mutter Sie wissen, dass Sie vieles gut können?") und Konflikte mit der Mutter (3 Items; Bsp.: Wie oft streiten Sie und Ihre Mutter miteinander?").

Tabelle 1: Hierarchische Regressionen zu den Effekten von Kultur, Normen und Werten sowie der Beziehungsqualität auf die Unterstützungsleistungen erwachsener Töchter für ihre Eltern (standardisierte Regressionskoeffizienten)

\begin{tabular}{|c|c|c|c|c|c|c|c|c|c|}
\hline \multirow[b]{2}{*}{ Korea $^{a}$} & \multicolumn{3}{|c|}{$\begin{array}{l}\text { Emotionale } \\
\text { Unterstutzung }\end{array}$} & \multicolumn{3}{|c|}{$\begin{array}{l}\text { Instrumentelle } \\
\text { Unterstultzung }\end{array}$} & \multicolumn{3}{|c|}{$\begin{array}{l}\text { Finanzielle } \\
\text { Unterstutzung }\end{array}$} \\
\hline & .01 & -.01 & .08 & $.16^{*}$ & .11 & $.20^{* *}$ & $.54^{* * *}$ & $.52 * *$ & $.56 * *$ \\
\hline Chin $^{a}{ }^{a}$ & $.25^{* *}$ & $.17^{*}$ & $.32^{* *}$ & $.29^{* *}$ & $.17^{*}$ & $.29^{* *}$ & $.50^{* *}$ & $.43^{* *}$ & $.54^{* *}$ \\
\hline Interdependenz & & $.20^{* *}$ & $.13^{*}$ & & $.17^{* * *}$ & $.12^{*}$ & & .07 & .05 \\
\hline $\begin{array}{l}\text { Filiale } \\
\text { Erwartungen }\end{array}$ & & .05 & -.02 & & $.15^{* *}$ & $.12^{*}$ & & $.10+$ & .08 \\
\hline $\begin{array}{l}\text { Konflikte } \\
\text { mit der Mutter }\end{array}$ & & & .08 & & & -.06 & & & .09 \\
\hline $\begin{array}{l}\text { Intimităt } \\
\text { zur Mutter }\end{array}$ & & & $.31^{* *}$ & & & $.10^{*}$ & & & .01 \\
\hline $\begin{array}{l}\text { Wertschătzung } \\
\text { durch Mutter }\end{array}$ & & & $.16^{* *}$ & & & $.24^{* *}$ & & & $.15^{* *}$ \\
\hline $\mathrm{R}^{2}$ & .07 & .11 & .26 & .15 & .20 & .28 & .26 & .28 & .30 \\
\hline
\end{tabular}

Datenbasis: VOC-Studie 2002

Anmerkung. Alle Auswertungen sind kontrolliert für Alter, Berufstätigkeit und Kinderzahl der Tochter sowie räumliche Distanz zu den Eltern.

${ }^{a}$ Referenzkategorie der Dummy-Variablen ist Deutschland.

${ }^{*} p<.05,{ }^{* *} p<.01$.

Wie Tabelle 1 zeigt, gehen eine höhere Intimität in der Beziehung zur Mutter und höhere Wertschätzung der Tochter durch die Mutter mit mehr emotionaler und instrumenteller Unterstützung der Töchter an die Eltern einher. Die Wertschätzung hängt auch mit der finanziellen Unterstützung durch die Tochter positiv zusammen. Die positive Qualität der Beziehung aus Sicht der Töchter trägt demnach über die Kulturen hinweg zu einer häufigeren Unterstützung bei. Konflikte scheinen dagegen eher weniger bedeutsam für die Unterstützungsleistung. 
Weiter wurden Regressionen zur Vorhersage der Häufigkeit der Unterstützung durch die Mütter berechnet. Es wurden dabei Intimität zur Tochter, Wertschätzung durch die Tochter und Konflikte mit ihr aus Sicht der Mütter einbezogen. Intimität in der Beziehung ging mit mehr emotionaler und finanzieller Unterstutzung an die Tochter einher, Wertschätzung der Mutter durch die Tochter hing positiv mit emotionaler Unterstützung zusammen. Anders als bei den Töchtern gingen Konflikte aus Sicht der Mutter über alle drei Kulturen hinweg einher mit mehr Unterstützung jeglicher Art. Die Frage, ob Konflikte zu mehr Unterstützung fuhren, um durch Zuwendung Konflikte zu kompensieren oder zu lösen, oder ob umgekehrt intergenerationale Unterstützung auch Anlass von Konflikten sein kann bzw. durch den häufigen Kontakt, der mit der Unterstützung verbunden ist, sich mehr Gelegenheiten für Konflikte ergeben, kann hier nicht beantwortet werden. Insgesamt lässt sich festhalten, dass neben den familienorientierten Normen und Werten kulturübergreifend auch die Beziehungsqualităt enge Zusammenhänge mit dem intergenerationalen Austausch von Unterstützung aufweist.

\subsection{Die kulturspezifische Bedeutung von Reziprozität}

Im Anschluss an die Fragen zum intergenerationalen Austausch von Unterstützung wurden die Töchter zu ihrer subjektiven Einschätzung der Reziprozität in diesem Austausch befragt: „Alles in allem, haben Sie das Gefühl, dass Sie Ihren Eltern mehr geben als Sie bekommen, ist es umgekehrt oder ist es ausgeglichen?" “. ${ }^{4}$ Die kulturspezifische Bedeutung, die Reziprozităt im intergenerationalen Austausch von Unterstützung hat, wurde aufgrund der Zusammenhänge mit der Beziehungsqualität sowie mittels verschiedener Indikatoren von Reziprozität und deren Zusammenhängen mit der Bereitschaft, in Zukunft die Eltern zu unterstützen, untersucht. Wieder wurden Stichproben aus Korea, China und Deutschland in die Vergleiche einbezogen.

Im Vergleich der ostasiatischen und deutschen Töchter fällt zunächst die signifikant unterschiedliche Verteilung auf die drei Kategorien der perzipierten Reziprozität auf (Tochter gibt mehr als sie erhält; ausgeglichen; Tochter erhält mehr als sie gibt). Während in der deutschen Stichprobe eine große Mehrheit

Im Folgenden werden nur Analysen zu den Angaben der erwachsenen Töchter aus der Valueof-Children Studie einbezogen und zwar fur die Gesamtgruppe von Frauen mit wenigstens einem Kind im Jugendalter. 
den Austausch von Unterstützung mit den Eltern als ausgeglichen bewertet (72\%), findet sich in der koreanischen Stichprobe nahezu eine Gleichverteilung auf die drei Kategorien (Tochter gibt mehr: $41 \%$, ausgeglichen: 27\%, Tochter erhält mehr: $38 \%$ ) und in der chinesischen eine Mehrheit, die glaubt mehr zu geben als zu erhalten (60\%) (Schwarz \& Trommsdorff 2004). Dieser Befund ist ein erster Hinweis darauf, dass der Druck, reziproke Verhältnisse in den Generationenbeziehungen herzustellen, in einer westlichen Kultur wie Deutschland größer ist als in den konfuzianischen Kulturen mit ihrer auch Generationen übergreifenden Langzeitorientierung (Hwang 1999).

Die Zusammenhänge mit der Beziehungsqualität verstärken den Eindruck der hohen Bedeutung der Reziprozitätsnorm in Deutschland für die Eltern-KindBeziehung im Erwachsenenalter. Berechnet wurden dazu hierarchische Regressionen, bei denen zunächst Kontrollvariablen, Kultur und die wahrgenommene Reziprozität und in einem letzten Schritt Interaktionsterme von Kultur und wahrgenommener Reziprozität eingeführt wurden, um Intimität, Konflikte und Wertschätzung in der Beziehung zur Mutter vorherzusagen. Für alle drei Indikatoren der Beziehungsqualität ergeben sich signifikante Interaktionen, die darauf hinweisen, dass sich die chinesischen und koreanischen Töchter signifikant von den deutschen Töchtern unterscheiden.

Die Wahrnehmung fehlender Reziprozität hat vor allem für die deutschen Töchter negative Folgen. Insbesondere, wenn sie wahrnehmen, dass sie mehr geben als sie von den Eltern bekommen, beschreiben sie die Beziehung zur Mutter als weniger intim, fühlen sich weniger von der Mutter wertgeschätzt und haben häufiger Konflikte mit ihr. Diese Zusammenhänge finden sich bei den koreanischen und chinesischen Töchtern nicht (für den Korea-Deutschland Vergleich siehe auch Schwarz, Trommsdorff, Kim \& Park, in press). In Abbildung 2 werden die signifikant unterschiedlichen Zusammenhänge der wahrgenommenen Reziprozität (hier der Vergleich Reziprozität vs. Tochter gibt mehr) mit der Intimität und den Konflikten in der Beziehung zur Mutter für die drei Kulturen dargestellt. Es zeigt sich, dass die Zusammenhänge für die koreanischen Töchter jeweils um Null liegen. Bei den chinesischen Töchtern finden sich ebenfalls kein Zusammenhang zwischen wahrgenommener Reziprozität und Intimität in der Beziehung und ein geringer positiver, jedoch nicht signifikanter Zusammenhang zwischen wahrgenommener Reziprozität und Konflikten. Nicht dargestellt sind die Befunde für die Wertschätzung. Genau umgekehrt zu den Befunden bei den deutschen Töchtern zeigt sich für die koreanischen und chinesischen Töchter, dass jene, die meinen, mehr zu geben als sie zurückbe- 
kommen, von mehr Wertschätzung durch die Mutter berichten (Korea $\beta=.16$, n. s.; China $\beta=.32, p<.05)$.

Abbildung 2: Die Qualität der Beziehung zur Mutter in Abhängigkeit von der durch die Töchter wahrgenommenen Reziprozität

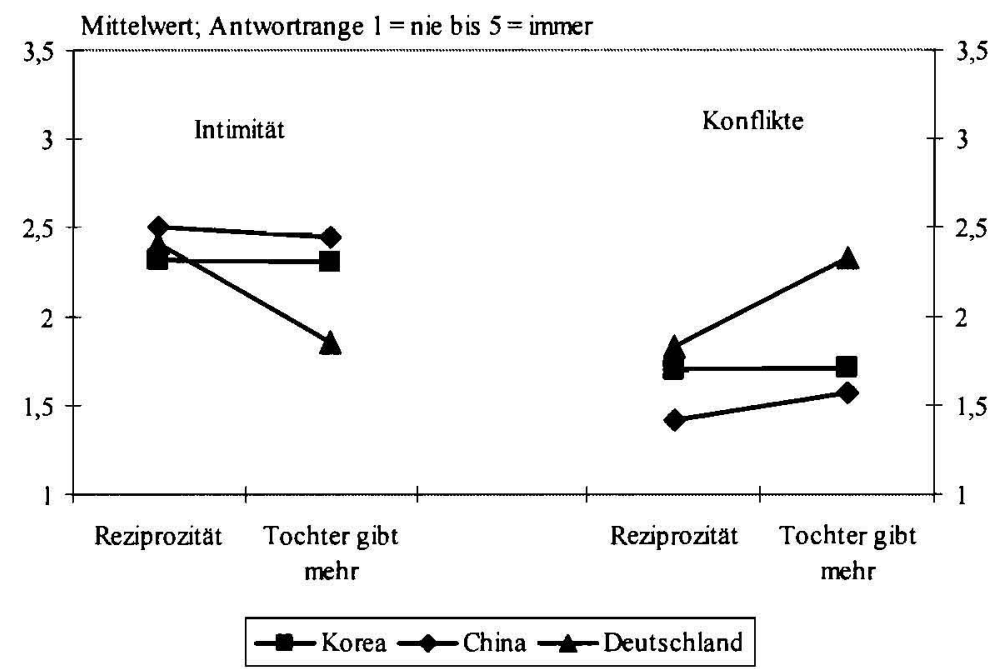

Datenbasis

Der Vergleich der koreanischen, chinesischen und deutschen Töchter in Hinblick auf die Zusammenhänge zwischen Reziprozität und ihrer Bereitschaft, in Zukunft einem pflegebedürftigen Elternteil zu helfen, unterstreichen diese Interpretation zusätzlich (Schwarz \& Trommsdorff 2004). Die Annahme war, dass mangelnde Reziprozität, insbesondere in Bezug auf mehr erfahrene Unterstützung durch die Eltern, dazu führt, dass die Tochter zumindest für die Zukunft eine Wiedergutmachung vorsehen, indem sie planen, besonders viele Belastungen auf sich zu nehmen, die im Zuge der Pflege der Eltern auftreten könnten. Die Wirksamkeit der Reziprozitätsnorm wurde in verschiedenen Facetten erfasst. Zum einen wurde wieder die subjektive Einschätzung der Reziprozität verwendet. Zudem wurde das Ausmaß an Unterstützung, die die Eltern geben, in die Analysen aufgenommen, da anzunehmen war, dass je mehr Unterstützung die Töchter aktuell bekommen, sie umso mehr Belastungen auf sich zu nehmen 
planen. Weiterhin wurde die Zustimmung zur Reziprozitätsnorm erfasst als Motivation, die bislang erfahrene elterliche Unterstützung durch spätere Pflegeleistungen zu kompensieren.

Die Ergebnisse zeigen durchgehend, dass die Reziprozitätsnorm für die deutschen Töchter eine sehr viel höhere Relevanz für ihre zukünftige Planung hat als für die koreanischen und chinesischen Töchter. Wenn die deutschen Töchter wahrnehmen, dass sie von den Eltern mehr Unterstutzung erhalten als sie bisher zurückgegeben haben, so planen sie, später mehr Belastungen für die Pflege der alten Eltern auf sich zu nehmen im Vergleich zu einer als ausgeglichen wahrgenommenen Bilanz von Geben und Nehmen. Je mehr Unterstützung die Eltern geben, desto stärker sind die deutschen Töchter bereit, spätere (Pflege-)Belastungen für die Eltern auf sich zu nehmen. Und je höher die Zustimmung der Töchter zur Reziprozitätsnorm ist, desto höher ist ihre geplante Belastungsbereitschaft. Die Zusammenhänge für die koreanischen und chinesischen Töchter sind dagegen alle nahe Null (Schwarz \& Trommsdorff 2004).

\section{Zusammenfassung und Ausblick}

Zusammenfassend verweisen die Befunde darauf, dass in den konfuzianisch orientierten Kulturen eine langfristigere Generationen übergreifende Zeitorientierung und die Möglichkeit des Abtragens von Schulden durch hohe Investitionen in die nächste Generation bewirkt, dass eine mangelnde Balance im intergenerationalen Austausch von Unterstützung weniger negative Folgen für die Eltern-Kind-Beziehung hat, und dass sich die erwachsenen Töchter weniger unter Druck fühlen, durch hohe zukünftige Pflegeleistungen für die alten Eltern eine Balance wieder herzustellen.

Für diese Interpretation spricht, dass die Befunde aus Korea und China sehr ähnlich sind und sich von den Ergebnissen der deutschen Stichprobe deutlich unterscheiden. Bemerkenswert ist dabei auch, dass konfuzianische Werte in den beiden ostasiatischen Kulturen trotz der bestehenden starken politischen und ökonomischen Unterschiede offenbar wirksam sind und zwar in ganz ähnlicher Weise. Auch wenn in Korea durch die rapide Industrialisierung und in der VR China durch die Kulturrevolution und die nachfolgenden massiven sozialen ökonomischen und politischen Änderungen der Konfuzianismus als alltagspraktische Handlungsorientierung in seiner Bedeutung abgenommen haben sollte, sind doch die dem Konfuzianismus zugrunde liegenden Familienwerte, durch die die Generationen einer Familie als miteinander verbundene System erlebt 
werden, für die Eltern-Kind-Beziehung weiterhin wirksam. Damit ist das Konzept der Reziprozität wie es aus klassischen austauschtheoretischen Ansätzen bekannt ist und auch so von Bengtson als wichtiges Element seines Modells intergenerationaler Solidarität verwendet wird, unter Einbeziehung indigener Kulturkonzepte wie sie sich auf den Konfuzianismus und der ,filial piety' ergeben, zu erweitern. Auf diese Weise können kulturvergleichende Studien einen Beitrag zu einer Differenzierung soziologischer Theorien leisten. Für die Interpretation von Befunden aus kulturvergleichenden Studien ist die Berücksichtigung kulturspezifischer Konzepte unerlässlich.

\section{Literatur}

Alt, Christian (1994): Reziprozităt von Eltern-Kind-Beziehungen in Mehrgenerationennetzwerken. In: Bien, Walter (Hrsg.): Eigeninteresse oder Solidarităt. Beziehungen in modernen Mehrgenerationenfamilien. Opladen: Leske + Budrich: 197-222.

Antonucci, Toni C. (1985): Personal Characteristics, Social Support, and Social Behavior. In: Binstock, Robert H. \& Shanas, Ethel (Eds.): Handbook of Aging and the Social Sciences. New York: Van Nostrand Reinhold Company: 94-128.

Bengtson, Vern L. (2001): Beyond the Nuclear Family: The Increasing Importance of Multigenerational Bonds. In: Journal of Marriage and the Family 63: 1-16.

Bengtson, Vern L. \& Roberts, Robert E. L. (1991): Intergenerational Solidarity in Aging Families: An Example of Formal Theory Construction. In: Journal of Marriage and the Family 53: 856870.

Bundesministerium fur Familie, Senioren, Frauen und Jugend (200I): Dritter Bericht zur Lage der alteren Generation in der Bundesrepublik Deutschland: Alter und Gesellschaft. Berlin: BMFSFJ.

Gouldner, Alvin W. (1960): The Norm of Reciprocity: A Preliminary Statement. In: American Sociological Review 25: 161-178.

Hwang, Kwang-Kuo (1999): Filial Piety and Loyalty: Two Types of Social Identification in Confucianism. In: Asian Journal of Social Psychology 2: 163-183.

Kohli, Martin \& Kunemund, Harald (2001): Geben und Nehmen. Die Älteren im Generationenverhältnis. In: Zeitschrift für Erziehungswissenschaft 4: 513-528.

Lauterbach, Wolfgang (1995): Die gemeinsame Lebenszeit von Familiengenerationen. In: Zeitschrift fur Soziologie 24: 22-43.

Lauterbach, Wolfgang \& Pillemer, Karl (2001): Social Structure and the Family: A United States Germany Comparison of Residential Proximity Between Parents and Adult Children. In: Zeitschrift für Familienforschung 13: 68-88.

Levande, Diane I./Herrick, John M. \& Sung, Kyu-Taik (2000): Eldercare in the Unites States and South Korea: Balancing Family and Community Support. In: Journal of Family Issues 21 : $632-651$.

Lye, Diane N. (1996): Adult Child-Parent Relationships. In: Annual Review of Sociology 22: 79102.

Mancini, Jay A. \& Blieszner, Rosemary (1989): Aging Parents and Adult Children: Research Themes in Intergenerational Relations. In: Journal of Marriage and the Family 51: 275-290.

Markus, Hazel Rose \& Kitayama, Shinobu (1991): Culture and the Self: Implications for Cognition, Emotion, and Motivation. In: Psychological Review 98: 224-253. 
Mayer, Boris/Albert, Isabelle/Schwarz, Beate \& Trommsdorff, Gisela (2003): Value-of-Children in Six Cultures: Item-Statistics, Reliability Analysis, Indicators. German Sample. Unveroffentlichter Bericht. Universität Konstanz.

Nauck, Bernhard (2001): Der Wert von Kindern fur ihre Eltern. "Value of Children" als spezielle Handlungstheorie des generativen Verhaltens und von Generationenbeziehungen im interkulturellen Vergleich. In: Kolner Zeitschrift für Soziologie und Sozialpsychologie 53: 407-435.

Nauck, Bernhard \& Suckow, Jana (2003): Generationenbeziehungen im Kulturvergleich. Beziehungen zwischen Muttern und Großmuttern in Japan, Korea, China, Indonesien, Israel, Deutschland und der Türkei. In: Feldhaus, Michael/Logemann, Niels \& Schlegel, Monika (Hrsg.): Blickrichtung Familie. Viel falt eines Forschungsgegenstandes. Wurzburg: Ergon: 5 I-66.

Schulz, Reiner (1996): Die Reziprozitat als konstitutives Netzwerkmerkmal. In: Zeitschrift für Bevölkerungswissenschaft 21: 263-280.

Schwarz, Beate/Chakkarath, Pradeep/Trommsdorff, Gisela/Schwenk, Otto \& Nauck, Bernhard (2001): Report on Selected Instruments of the Value-of-Children Study (Main Study). Unveroffentlichter Bericht. Universitat Konstanz.

Schwarz, Beate \& Trommsdorff, Gisela (2004): Reciprocity in Intergenerational Support: A Comparison of Korea, China, and Germany. Manuscript submitted for publication.

Schwarz, Beate/Trommsdorff, Gisela/Mayer, Boris \& Albert, Isabelle (2004, July). Intergenerational Support: A Comparison of Women from the Republic of Korea, China, and Germany. Paper presented at the 18. Biennial meeting of the International Society for the Study of Behavioral Development, Ghent, Belgium.

Schwarz, Beate/Trommsdorff, Gisela/Kim, Uichol \& Park, Young-Shin (in press): Intergenerational Support: Psychological and Cultural Analyses of Korean and German Women. In: Current Sociology.

Sun, Rongjun (2002): Old Age Support in Contemporary Urban China from Both Parents' and Children's Perspectives. In: Research on Aging 24: 337-359.

Szydlik, Marc (2000): Lebenslange Solidarität? Generationenbeziehungen zwischen erwachsenen Kindern und Eltern. Opladen: Leske + Budrich.

Trommsdorff, Gisela (2001): Eltern-Kind-Beziehungen aus kulturvergleichender Sicht. In: Walper, Sabine \& Pekrun, Reiner (Hrsg.): Familie und Entwicklung. Aktuelle Perspektiven der Familienpsychologie. Gottingen: Hogrefe: 36-62.

Trommsdorff, Gisela (2003): Kulturvergleichende Entwicklungspsychologie. In: Thomas, Alexander (Hrsg.): Kulturvergleichende Psychologie. 2. uberarbeitete Auflage. Göttingen: Hogrefe: 139-179.

Trommsdorff, Gisela (in press): Parent-Child Relations Over the Life-Span: A Cross-Cultural Perspective. In: Rubin, Kenneth H. (Ed.): Parental Beliefs, Parenting, and Child Development in Cross-Cultural Perspective. New York: Psychology Press.

Trommsdorff, Gisela \& Komadt, Hans-Joachim (2003): Parent-Child Relations in Cross-Cultural Perspective. In: Kuczynski, Leon (Ed.): Handbook of Dynamics in Parent-Child Relations. Thousand Oaks,CA: Sage: 27I-306. 